\title{
SEMT - Sensorische Erkennung der Materialart und Er- fassung der Trennflächen
}

\author{
PROF. DR.-ING. LUDGER OVERMEYER, \\ DIPL.-ING. MARTIN KESTING \\ INSTITUT FÜR TRANSPORT- UND AUTOMATISIERUNGSTECHNIK, LEIBNIZ UNIVERSITÄT HANNOVER
}

\section{Zusammenfassung}

Das Ziel des Projektes SEMT ist die Unterstützung der qualitätsrelevanten Gewinnung durch Erkennung der Materialarten und der dazwischen liegenden Trennfläche (Schichtgrenze) während des Grabvorganges als Geräteführerhilfe und zur Verfeinerung zukünftiger Tagebaumodelle. Die in Echtzeit erfolgende Erkennung des anstehenden Materials, der Trennflächen und die während des Grabvorgangs erfolgende Vorauserkundung in die Tiefe bilden die Basis für weitere Automatisierungsvorhaben und Prozessoptimierungen in der gesamten Abbaukette von Gewinnung, Transport, Lagerung und Verkippung. Zur Auswahl einer geeigneten Sensorik wurde eine Vorstudie mit anschließender Wertung in Bezug auf die Leistungsanforderungen und Einsatzbedingungen am Schaufelradbagger durchgeführt. Die aus dieser Studie als theoretisch geeignet erscheinenden Sensoriken Georadar und Geoelektrik mussten in Feldversuchen ihre praktische Eignung für SEMT nachweisen. Dieser Bericht gibt eine Übersicht über die durchgeführten Feldversuche mit den dazugehörigen Ergebnissen und einen Ausblick auf die mögliche Integration der Sensoriken am Schaufelradbagger.

\section{Abstract}

The efficiency of continuous mining technologies depends to a large degree on a flawless and synchronous operation of the complete process chain. From certain points, a further optimization of this operation can only be achieved by gathering additional information about the input parameter. The earlier and more exact the description of the input parameter, the greater the effectiveness of the entire process chain. The objective of the SIMT project is to support quality-relevant mining by the identification of the material types and the interface (layer border) lying between them during the mining process as an operator's aid, and for the refinement of future open mining models. The identification of the in-situ materials, interfaces in real-time, and the exploratory subsurface investigations which occur during the mining operation, form the basis for further automation projects and process optimisation in the entire mining chain of excavation, transport, storage and dumping. In order to select a suitable sensor technology a preliminary study was carried out with subsequent evaluation with regard to performance requirements and operating conditions of the bucket wheel excavator. Ground penetrating radar and geo electric, which, in theory, seemed suitable sensor technologies as a result of this study, had to prove their practical suitability for SIMT in field tests. In principle, two possibilities exist for the integration of the sensor technology. It can be integrated next to the bucket wheel or in the bucket wheel itself. For the ground penetrating radar both variants are possible since direct contact with the material is not necessary. The inducting and measuring electrodes of geo electric, however, must be integrated in the bucket, because direct contact with the in situ material is necessary. This paper gives an overview of the field tests carried out, with the corresponding results, and the future prospects of the possible integration of the sensor technologies on the bucket wheel excavator.

\section{Zukunftspotenzial durch SEMT}

Die kontinuierliche Gewinnung hat ihre Stärke in der Realisierung von großen Fördermengen während des Abbauvorgangs durch den Schaufelradbagger. Dieser Vorteil kommt jedoch nur dann zum Tragen, wenn die gesamte Prozesskette in der kontinuierlichen Gewinnungstechnik möglichst störungsfrei betrieben werden kann [DUR86]. Eine Unterbrechung innerhalb der Prozesskette hat einen Stopp des Schaufelradbaggers und der damit verbundenen Gewinnung zur Folge. Dieses zeigt sich auch in der Gesamtausnutzung der Bagger-Kapazität der RWE Power AG, welche sich im Jahr 2005 auf 42,8 \% belief (siehe Abb.1). Die Gesamtqualität jeder Prozesskette steigt im Besonderen mit der Kenntnis der Eingangsparameter. Je genauer und frühzeitiger sich die Eingangsparameter beschreiben lassen, umso größer ist die Effektivität der gesamten Prozesskette. Das primäre Potenzial des Forschungsvorhabens SEMT ist bei der Gewinnung zu sehen. 
Hier wird zunächst die manuelle Materialansprache in eine automatische Materialansprache und Trennflächenerkennung (Schichtgrenzenerkennung) überführt.

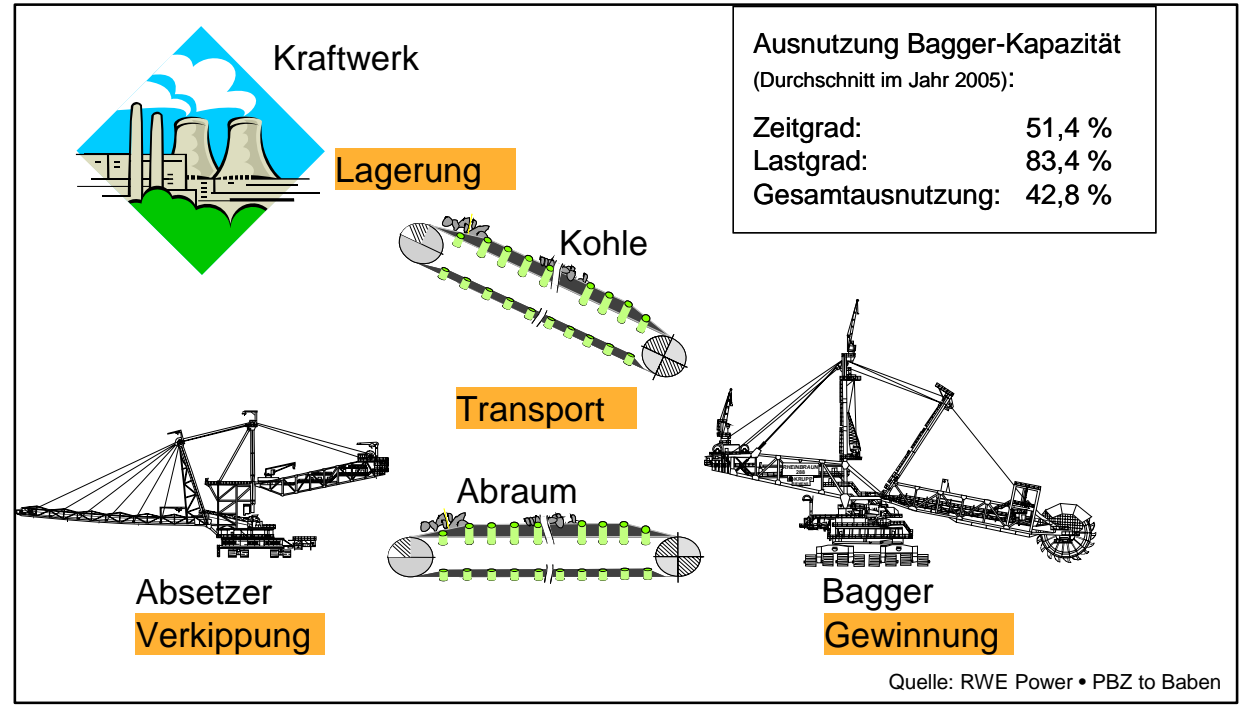

Abbildung 1: Prozesskette der kontinuierlichen Gewinnung im Braunkohlentagebau

Dadurch soll insbesondere die Genauigkeit der Materialansprache erhöht werden. Hierauf basierend kann dem Geräteführer ein Assistenzsystem zur Verfügung gestellt werden, welches eine abbauoptimierte Steuerung unter Berücksichtigung der folgenden Aspekte ermöglicht:

- $\quad$ Aushaltung von Materialarten

- Gezielte Erreichung von Mischungsverhältnissen

- Zeitnahe Anpassung der optimalen Abbauparameter

Die im Projekt SEMT gewonnenen Erkenntnisse bilden die Basis zur Erreichung der als Fernziel anzusehenden Vollautomatisierung des Schaufelradbaggers.

Weiterhin werden Beiträge zur Förderprozessoptimierung in den folgenden Bereichen geliefert:

- $\quad$ Steuerung der Bandanlagen

- $\quad$ Reduzierung von Emissionen und Verschleiß

- Optimierung der Verkippungsprozesse

- Höhere Auslastung der Absetzer

Das Vorhaben SEMT bietet ein hohes Potenzial zur Prozessoptimierung, welches aber nur mit einer entsprechend leistungsfähigen Sensorik erreicht werden kann.

\section{Geeignete und favorisierte Sensorik}

\subsection{Machbarkeitsstudie zur Auswahl einer geeigneten Sensorik}

Die Ergebnisse einer bereits durchgeführten Vorstudie zeigen, dass es zwar ein breites Spektrum an Sensoriken zur Material- und Trennflächenerkennung gibt, hiervon aber nur wenige zum Online-Messeinsatz am Schaufelradbagger geeignet sind. Unter Beachtung der Anforderungen an die Sensorik, der durchgeführten Studien und des bereits vergleichbar stattfindenden Einsatzes einzelner Sensoriken in der Praxis werden folgende Messverfahren favorisiert:

- Georadar, Reflexionsmessungen

- Geoelektrik, Widerstandsgeoelektrik

- $\quad$ induzierte Polarisation

- Bildverarbeitung, Thermographie

- $\quad$ LIF Laserinduzierte Fluoreszenz

- Infrarotspektroskopie 
Nur eine Kombination von Messverfahren zu einer Multisensorik wird die voraussichtlich benötigte Auflösung und Genauigkeit erreichen können. Unter Berücksichtigung der physikalischen, technischen und wirtschaftlichen Belange ist die Kombination aus Georadar und Geoelektrik theoretisch am besten geeignet. Georadar und Geoelektrik sind hierbei die einzigen Sensoriken, welche in den Untergrund eindringen und Informationen von unter der Oberfläche liegenden Materialarten und geologischen Schichtungen liefern.

\subsection{Georadar}

Das Georadarverfahren zählt zu den Impuls-Reflexionsverfahren. Über eine Sendeantenne werden kurze elektromagnetische Impulse in den Untergrund abgestrahlt. Diese elektromagnetischen Wellen breiten sich im Untergrund aus und werden an den Schichtgrenzen unterschiedlicher Materialarten teilweise reflektiert. Die Reflexionssignale werden von einer Empfängerantenne erfasst. Aus der gemessenen Laufzeitdifferenz zwischen Sendeund Empfangssignal können bei bekannter Ausbreitungsgeschwindigkeit der elektromagnetischen Welle im Untergrund nach entsprechender Signalbearbeitung auf die Teufenlage nachgewiesener Reflektoren wie z.B. Schichtgrenzen, Hohlräume und anthropogene Einlagerungen geschlossen werden. [ALT04, BER98, BRI03, DAN04 ]

Ein entscheidender Vorteil des Georadarverfahrens besteht darin, dass es sich bei dieser geophysikalischen Methodik um ein kontinuierliches bzw. quasikontinuierliches und damit sehr schnell messendes Verfahren handelt. Sende- und Empfangsantenne können kontinuierlich über das zu untersuchende Medium bewegt werden [FOER01]. Bestimmte Ankopplungsbedingungen der Antennen an das Gestein oder den Untergrund sind nicht erforderlich. Die Wahl der Aufzeichnungsgeschwindigkeit bestimmt damit die Messdichte der Untergrundabtastung. Abbildung 2 zeigt schematisch die Funktion eines Georadargerätes. Bei herkömmlichen Untersuchungen sind Messpunktdichten im Zentimeter- bis Meterbereich üblich. Mittels dieser zeit- und kosteneffizienten Messmethodik können somit räumliche Strukturinformationen zum geologischen und anthropogenen Aufbau des Untergrundes gewonnen werden. Die Georadartechnologie kann durch Nutzung konventioneller Oberflächenantennen im Frequenzbereich zwischen $10 \mathrm{MHz}$ und 2,5 GHz sowohl von der Erdoberfläche als auch im untertägigen Bergbau eingesetzt werden. Durch spezielle Maßnahmen in Form der Integration von stark dämpfenden Dielektrika in den Antennen kann eine mehr oder weniger gute Richtwirkung der abgestrahlten, elektromagnetischen Energie bei abgeschirmten Oberflächen-Antennen erzielt werden [DAN04 ]. Je höher die Frequenz der abgestrahlten Radarwellen ist, um so besser ist die räumliche Auflösung der zu untersuchenden Untergrundstrukturen. Begrenzende Größe für die Anwendung hochfrequenter Antennensysteme ist allerdings das mit zunehmender Frequenz einhergehende höhere Dämpfungsverhalten, sodass die erforderliche Reichweite zur Ortung von Schichtverläufen in Abhängigkeit vom Gestein physikalisch nur bis zu bestimmten Tiefen möglich ist. An einer Schicht- oder Materialgrenze kommt es zu einer Reflexion der elektromagnetischen Welle, wenn sich Materialien in ihren dielelektrischen Eigenschaften (Dielektrizitätskonstante, Hochfrequenzleitfähigkeit) voneinander unterscheiden. Je höher der petrophysikalische Kontrast dieser Parameter ist, desto höher ist der reflektierte Energieanteil und um so sicherer der Nachweis der Reflexionsgrenze [DAN04 ]. 


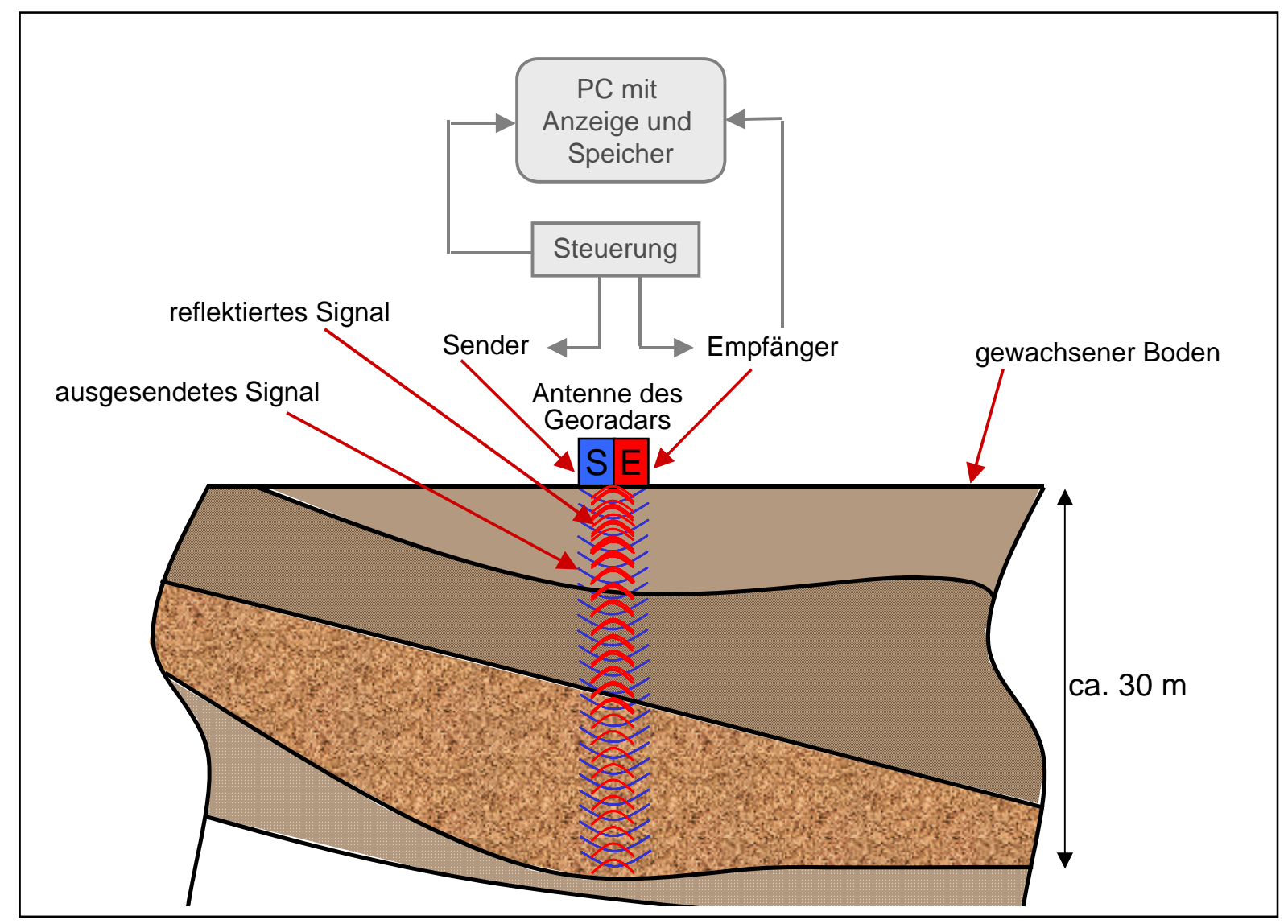

Abbildung 2: Funktionsprinzip Georadar

Die Wahl der für die spezifische Erkundungsaufgabe optimalen Antennenfrequenz ist dabei immer ein Kompromiss zwischen der geforderten Auflösung, z. B. im Nachweis kleindimensionaler Strukturen und den dielektrischen Eigenschaften der im Untersuchungsgebiet anstehenden Gesteine. Der Einsatz des Georadars im Reflexionsmodus in Gesteinen mit hoher elektrischer Leitfähigkeit ist nur für geringe Eindringtiefen möglich. In schluffigen, mergligen Lockersedimenten sowie in stark verwittertem Felsgestein treten gewöhnlich sehr hohe Dämpfungen der elektromagnetischen Wellen auf, welche die für die Erkundung erforderliche Tiefenreichweite stark begrenzen. Für die erzielbaren Erkundungsreichweiten können folgende allgemeingültige Richtwerte gegeben werden:

- Wenige Meter oder darunter in tonigen und schluffigen Gesteinen

- 10 bis 40 Meter in normal geklüfteten Fels- oder rolligen Sedimentgesteinen

- 40 bis 150 Meter in massiven Gesteinen

- $\quad$ bis zu 300 Metern und mehr bei Messungen unter speziellen Bedingungen (reines Steinsalz, ungestörter Kalkstein, Granit, Eis,...)

Die erforderlichen Erkundungsreichweiten für SEMT können direkt aus den maximalen Spantiefen während des Grabvorganges abgeleitet werden. Die Spantiefe beim Braunkohleabbau liegt zwischen 0 und ca. 1,80 m. Somit ist eine Vorauserkundung von ca. 2 m Eindringtiefe durch das Georadar ausreichend. Diese Eindringtiefe wird auch in für das Georadar ungünstigen Umgebungsbedingungen bzw. Materialarten erreicht. Ausschließlich an feuchten Tonböden erfolgt eine so starke Reflexion des Signals oder Absorption des eindringenden Restsignals, dass nur geringe Eindringtiefen möglich sind. Das Auftreten dieser Situation kann damit gleichzeitig sehr gut zur Detektion einer Tonschicht genutzt werden. Die durch die RWE Power AG gewünschte Auflösung von +/- 10 cm ist mit der entsprechenden Messfrequenz, Antennengeometrie und Anordnung erreichbar.

\subsection{Geoelektrik}

Ziel der Messungen ist es, die räumliche Verteilung des Widerstandes im Gebirge zu bestimmen. Bei den Widerstandsmessungen werden in den Untergrund über Punktelektroden Ströme eingespeist, um ein elektrisches Potenzialfeld aufzubauen. Mit weiteren Elektroden wird die erzeugte Potenzialfeldverteilung gemessen. 
Da der eingespeiste Strom, die Potenzialdifferenz zwischen den Messelektroden sowie die Elektrodenanordnung bekannt sind, kann der scheinbare Widerstand des Untergrundes ermittelt werden [SCH00, SIM05].

Widerstandsmessungen können sowohl von der Erdoberfläche aus als auch zwischen Bohrlöchern oder zwischen einem Bohrloch und der Oberfläche durchgeführt werden. Es sind Kartierungen, Sondierungen, 2D- und 3DMessungen sowie tomographische Messungen möglich. Als erstes Ergebnis aller widerstandsgeoelektrischen Messungen erhält man Informationen über die Verteilung des scheinbaren spezifischen Widerstandes. Das bedeutet, dass der gemessene Spannungswert von allen stromdurchflossenen Schichten und Objekten beeinflusst wird und auch von der eingesetzten Elektrodenkonfiguration abhängt. Die Verteilung der wahren spezifischen Widerstände wird mittels geeigneter Inversionsalgorithmen berechnet. Die Resultate sind quantitative Modelle des Untergrunds. Wie bei jedem Potenzialverfahren sind die Modelle keine eindeutigen Lösungen. Zur Beurteilung der Gültigkeit eines Inversionsergebnisses benötigt man Zusatzinformationen aus Aufschlüssen oder anderen geophysikalischen Ergebnissen. Die Widerstandsgeoeletrik eignet sich besonders zur Bestimmung des Feuchtegehalts der untersuchten Materialart. In Kombination mit der induzierten Polarisation ergibt sich eine gute Möglichkeit, die Materialart zu bestimmen und dabei auch zwischen trockenem, feuchtem und nassem Material zu unterscheiden. Die induzierte Polarisation charakterisiert die Eigenschaft des Untergrundes, elektrische Energie zu speichern. Dieses wird durch die unterschiedliche Mobilität von Ionen im Poren- und Kluftraum bewirkt, wenn ein elektrisches Feld angelegt wird. Die Membranpolarisation wird durch Ionentransportunterschiede verursacht, wenn dort, wo Poren- und Kluftspaltenhälse ausgebildet sind, elektrische Ladungen akkumuliert werden. Geologische Formationen mit kleinen Poren und engen Kluftspalten erzeugen höhere Polarisation als Gebiete mit großen Poren, weiter geöffneten Klüften und Karstholräumen [GEO04, KLI03].

\section{Integration der Sensorik am Schaufelradbagger}

Für die Integration der Sensoren bestehen grundsätzlich zwei Möglichkeiten: Sie können neben dem Schaufelrad oder in das Schaufelrad integriert werden. Abbildung 3 zeigt schematisch die mögliche Platzierung der Sensorik. Für die Sensorik Georadar kommen beide Varianten in Betracht, da kein direkter Kontakt zum zu untersuchenden Material erforderlich ist. Die einspeisenden und messenden Elektroden der Geoelektrik müssen dagegen in eine Schaufel integriert werden, weil ein direkter Kontakt zum anstehenden Material erforderlich ist.

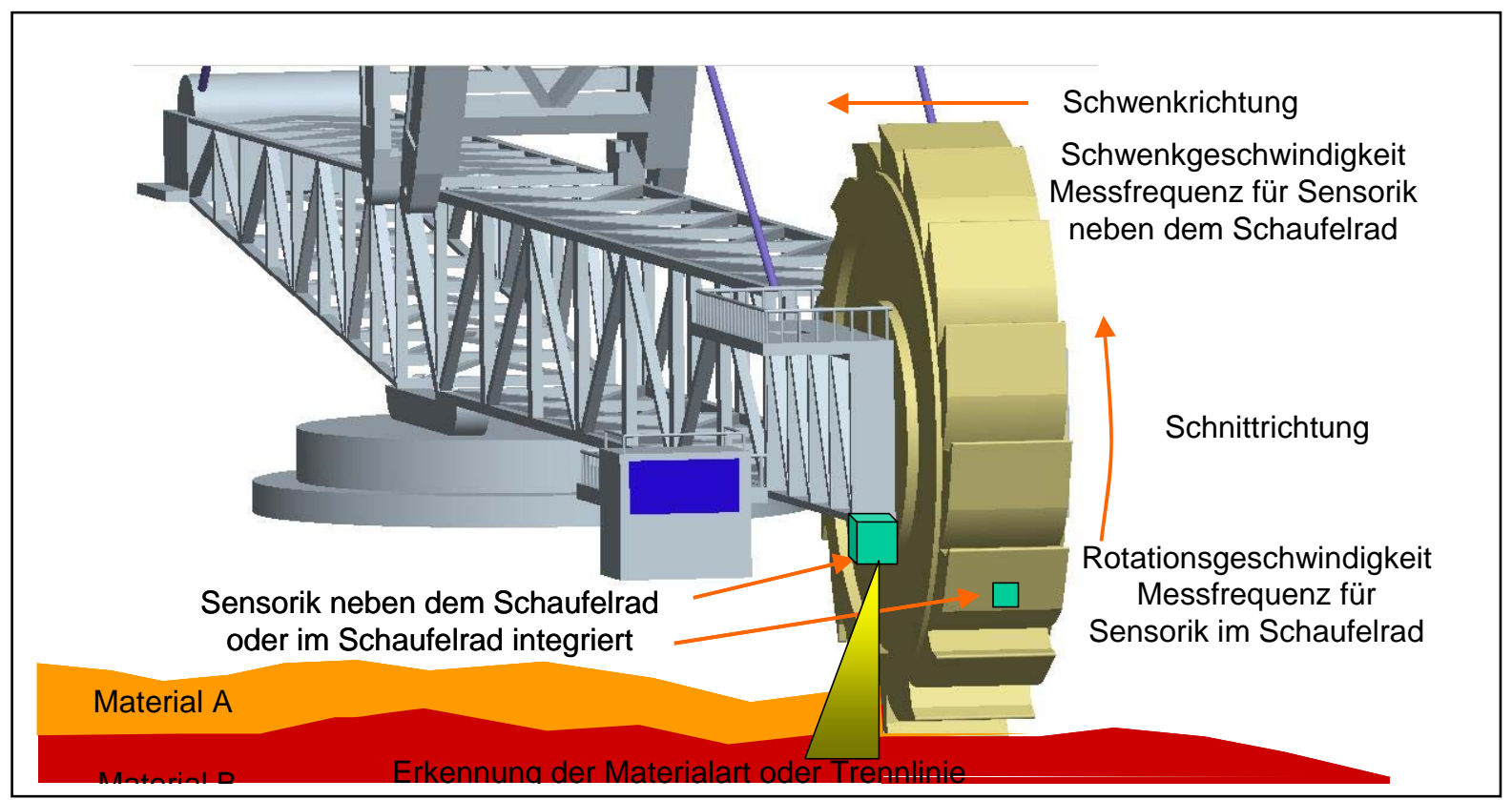

Abbildung 3: Platzierung der Sensoriken am Schaufelradbagger 


\section{Feldversuche}

Im ersten Meilenstein des SEMT-Forschungsvorhabens, welches im August 2006 endete, konnte der praktische Nachweis für die Sensoriken Georadar und Geoelektrik durch Feldversuche im Tagebau Inden der RWE Power AG erbracht werden. In den folgenden Absätzen wird die Vorgehensweise bei den Feldversuchen näher beschrieben.

\subsection{Versuchsaufbau und Versuchsdurchführung}

Für die Feldversuche mit Georadar und Geoelektrik wurden von der RWE Power AG im Tagbau Inden Versuchsflächen in der Ebene und am Abbaustoß mit unterschiedlichem geologischem Aufbau der Materialschichtung und dem anstehendem Material zur Verfügung gestellt.

\subsubsection{Georadar}

Für das Georadar wurden unterschiedliche Versuchsaufbauten gewählt, um die Situationen der Platzierung am Schaufelradbagger zu simulieren. Die Bilder in Abbildung 4 zeigen die verwendeten Aufbauten zur Versuchsdurchführung auf den ebenen Versuchsflächen, welche im ersten Schritt für eine generelle Untersuchung zur Verwendbarkeit der durch Georadar gewonnenen Messdaten genutzt wurden. Auf dem ersten Bild wurden die Antennen in einen Metallschlitten integriert, um somit die Platzierung auf dem Schaufelrücken nachzubilden. Bild 2 zeigt den Versuchsaufbau, mit dem die Antennen mit unterschiedlichem Abstand über die Messoberfläche geführt wurden, um so die Auswirkungen von verschiedenen Offsetabständen zu erfassen. Weiterhin konnte mithilfe eines Schwenkantriebs der Antennenwinkel zum Boden verändert werden. Mit der in Bild 3 gezeigten Versuchsanordnung wurden die Referenzmessungen aufgenommen. Durch die Platzierung der Antennen auf einen Holzschlitten konnten Umgebungsbeeinflussungen auf die Antenne auf ein Minimum reduziert werden.

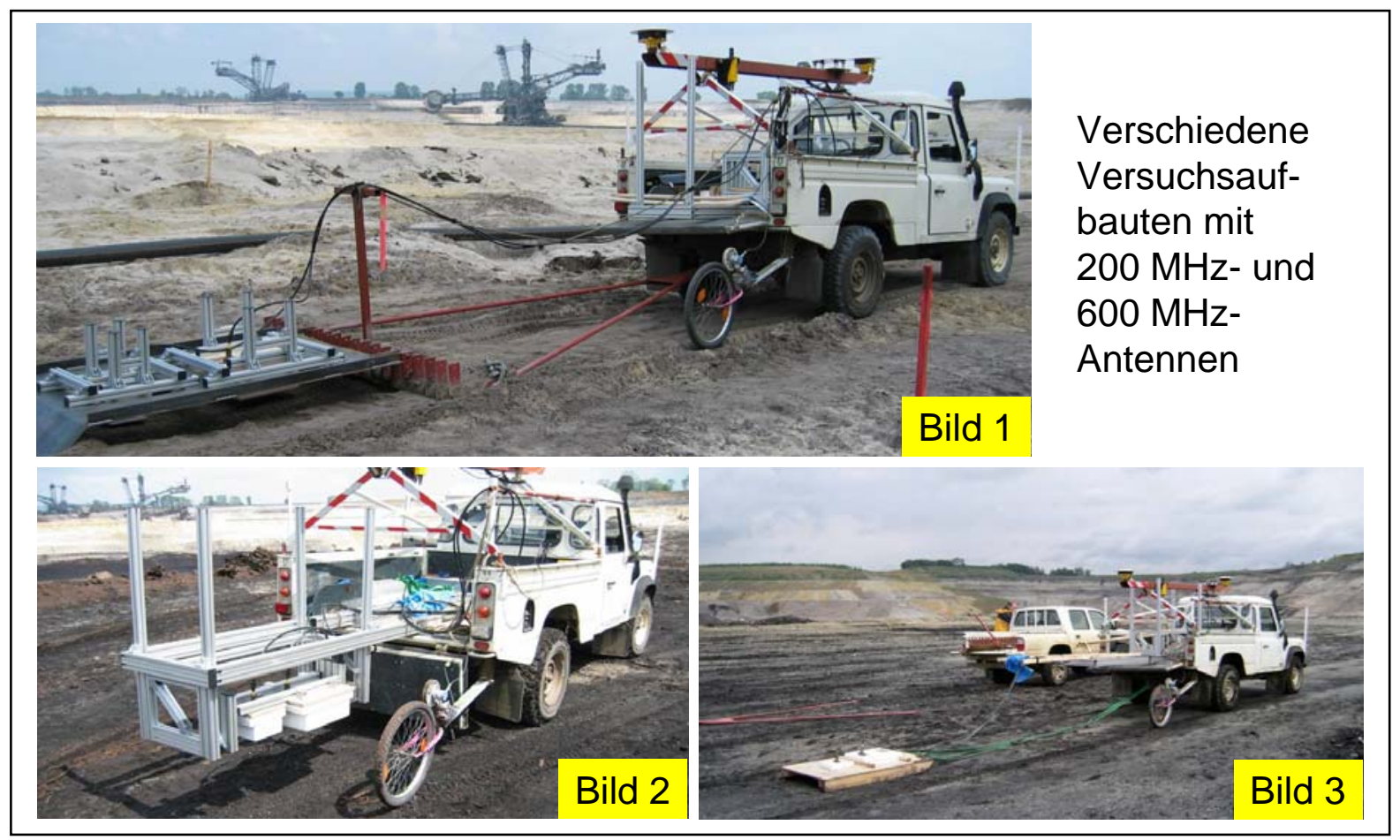

Abbildung 4: Georadarversuche in der Ebene

Die Verknüpfung der gewonnenen Messdaten mit der absoluten räumlichen Lage erfolgte auf zwei Wegen:

1. Genaue Einmessung der Versuchsflächen in Kombination mit einem Messrad, welches hinter dem Versuchsfahrzeug hergezogen wurde.

2. Mithilfe eines hochgenauen Differenzial-GPS-Systems mit einer Messgenauigkeit der absoluten räumlichen Lage von $+/-3 \mathrm{~cm}$.

Abbildung 5 zeigt ein in der Ebene aufgenommenes Radargramm, aus dem der Verlauf der Schichtgrenze zwischen Sand und Kohle deutlich ersichtlich ist. Nach Abschluss der Radarmessungen wurden einzelne Versuchsflächen aufgegraben und die Schichtgrenzen markscheiderisch aufgemessen. Die gelben Markierungen zeigen das markscheiderische Aufmaß, welches mit dem Radargramm übereinstimmt, womit eine hohe Genauigkeit des Radargramms nachgewiesen ist. 
Vergleicht man den realen Schichtgrenzverlauf aus dem Radargramm und dem markscheiderischen Aufmaß mit den Daten des Lagerstättenbaumodells, ergeben sich in diesem Beispiel Abweichungen von 4 bis 5 m. Diese Abweichungen resultieren aus dem groben Raster der Vorerkundungsbohrungen und der Interpolation zwischen diesen Bohrungen. Diese Sachlage unterstützt die Forderung nach einer Online-Erfassung der Trennflächen am Schaufelradbagger mit automatischer Visualisierung im Führerstand und der Einbringung der Daten in die Bergbauplanungsvorgänge als Basis für eine weitere Steigerung der Förderqualität und Automatisierung.

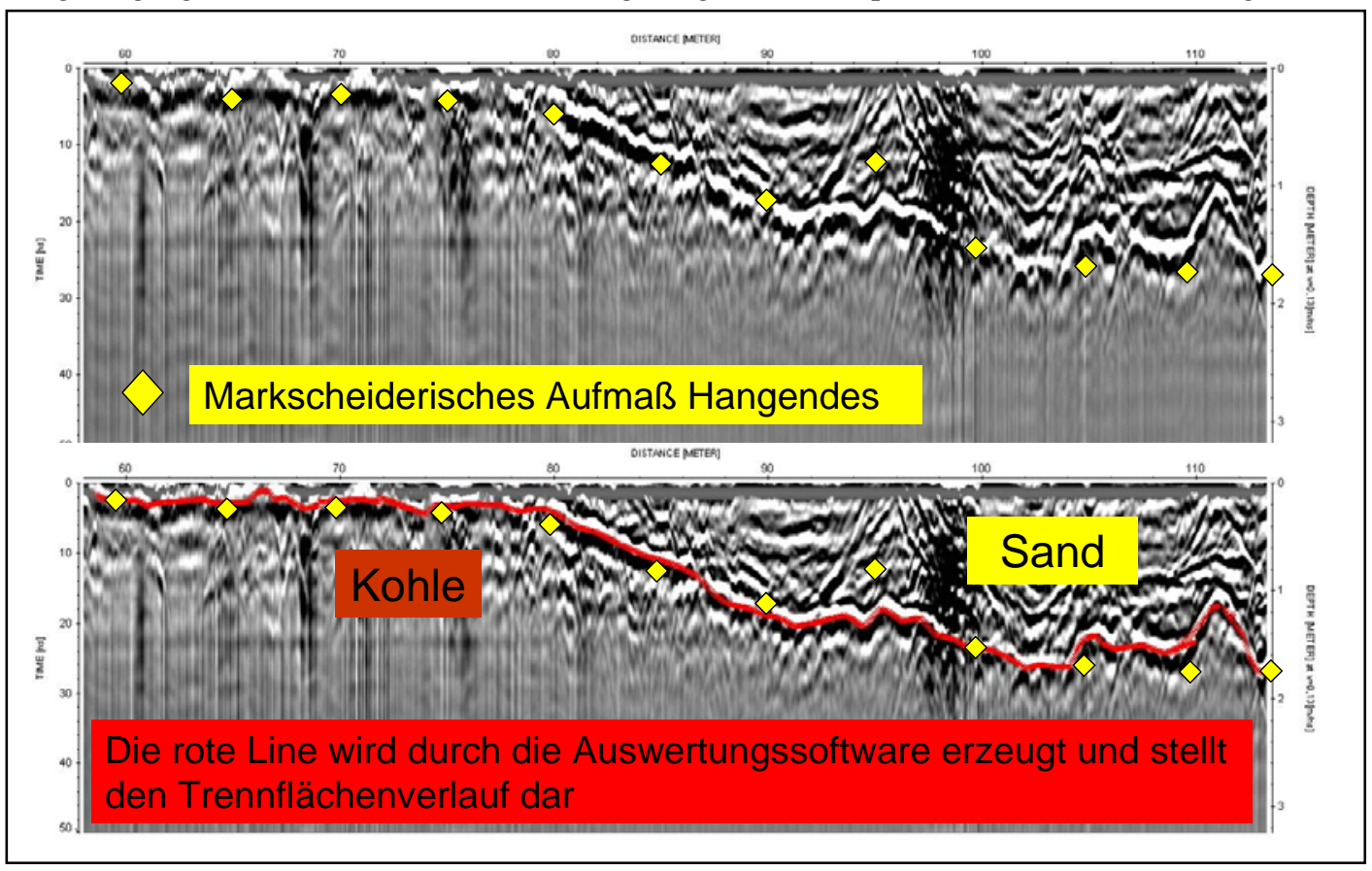

Abbildung 5: Radargramm einer stärker werdenden Sandschicht über Kohle

Im zweiten Schritt wurden die Versuche nicht mehr in der Ebene, sondern, wie in Abbildung 6 dargestellt, am Abbaustoß durchgeführt.

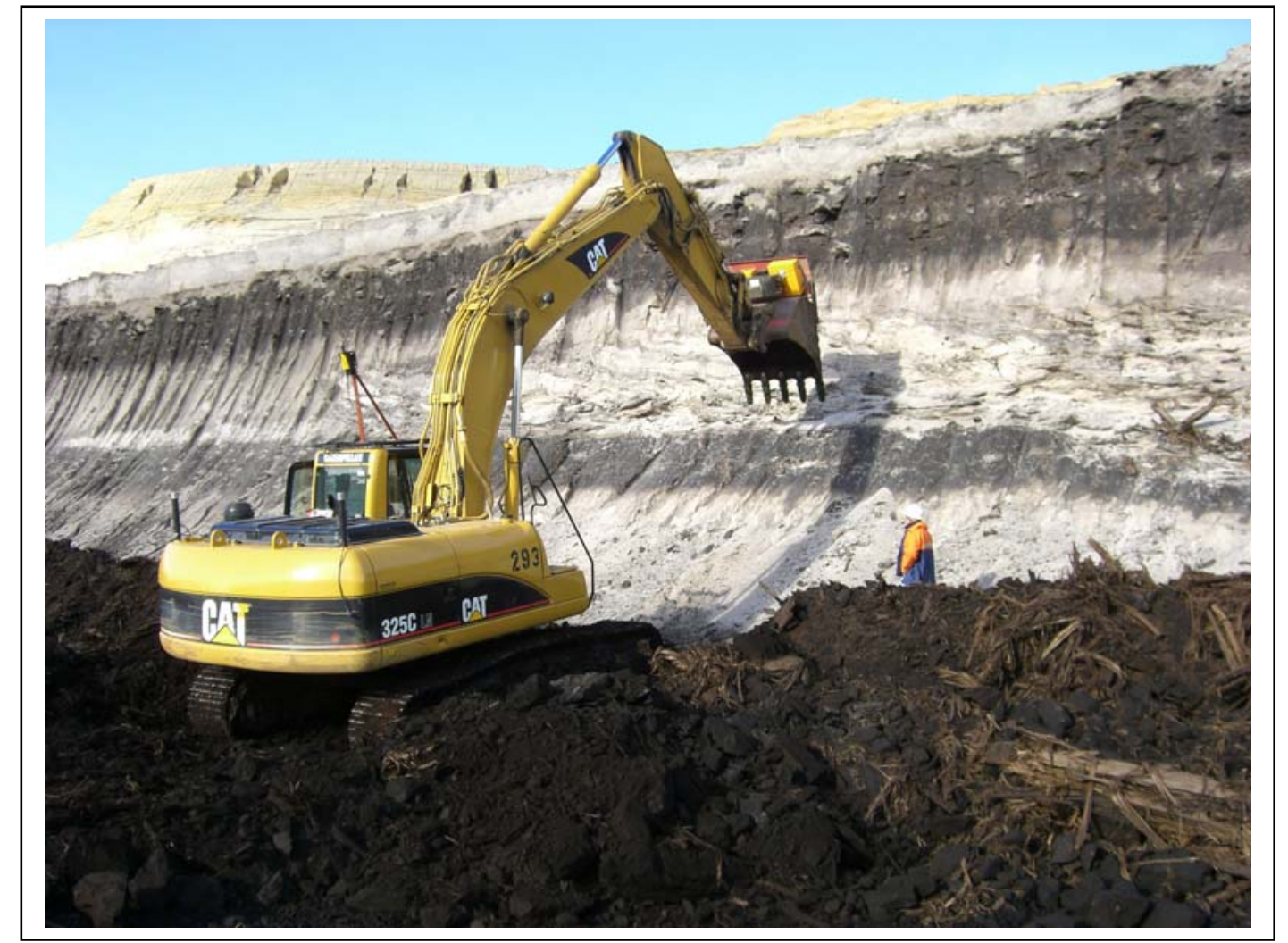

Abbildung 6: Georadarversuche am Abbaustoß 
Die Sensorik wurde auf der Rückseite einer Hydraulikbaggerschaufel platziert und konnte so variabel entlang der Abbaustoßgeometrie geführt werden. Mit diesem Aufbau konnte die spätere Führung der Georadarantenne am Schaufelradbagger nachgebildet werden. Mithilfe einer Trimble Totalstation konnte der Positionsverlauf der Georadarsensorik im dreidimensionalen Raum erfasst werden. Hierzu wurde an der Antennenhalterung auf dem Schaufelrücken des Hydraulikbaggers ein Prisma befestigt, welches kontinuierlich vom Laser der Totalstation verfolgt wurde. In Abbildung 7 ist die örtliche Messsituation eines Kohleflözes, welches eine Ton-/Sandschicht überlagert, dargestellt. Das dazugehörige Radargramm zeigt dabei die Schichtdicke des Kohleflözes bis zur Schichtgrenze der Ton-/ Sandschicht an. Der reale Grenzschichtverlauf kann später über die Antennenposition und Winkelstellung des Schaufelrades berechnet werden.

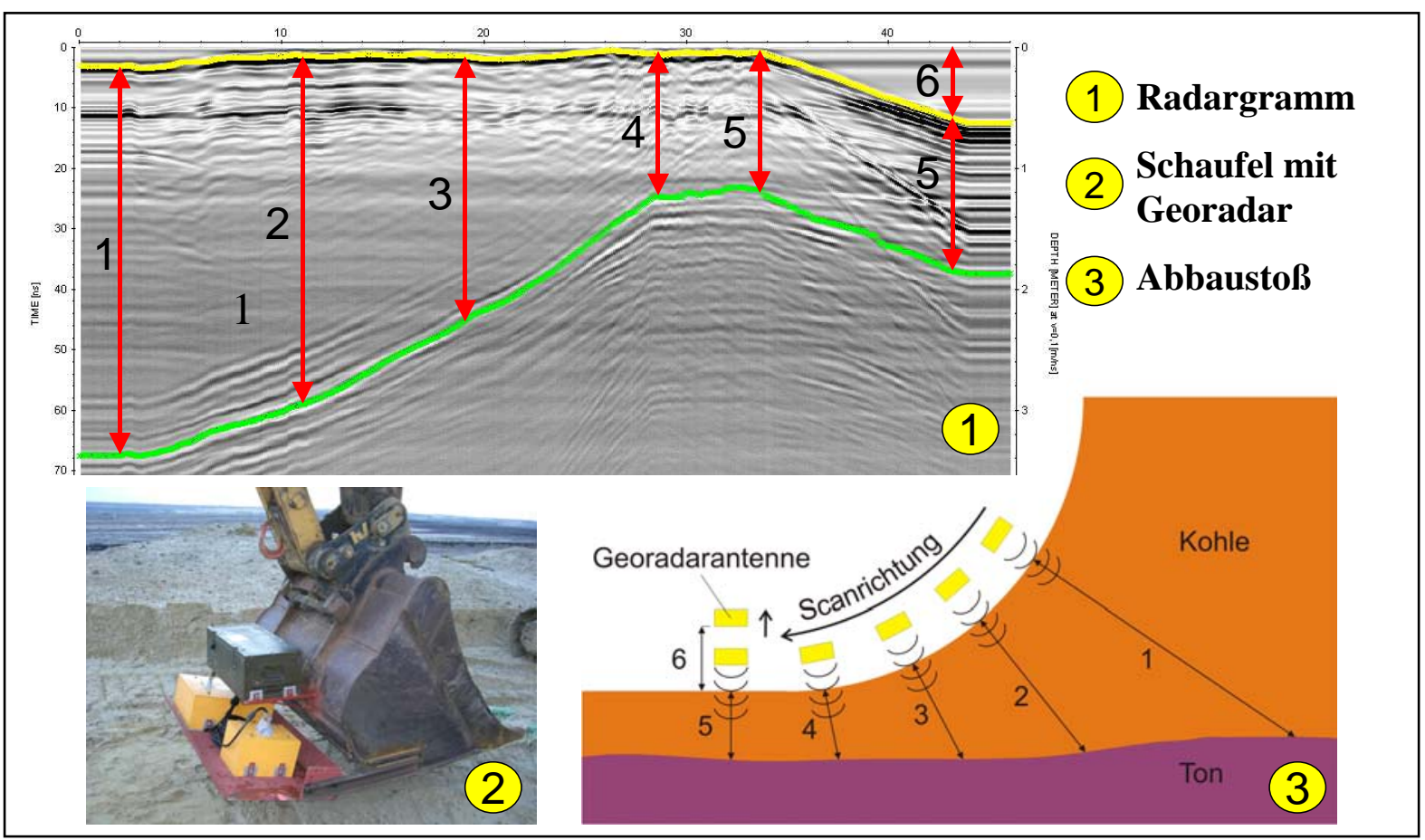

Abbildung 7: Georadarmessungen am Abbaustoß 


\subsubsection{Geoelektrik}

Geoelektrische Messungen erfordern den direkten Kontakt zum Untergrund. Für die Feldversuche wurden spezielle Messkufen entwickelt, um die spätere Integration von Elektroden in die Schaufelkante zu simulieren. Mit den Messkufen wurden wie in Abbildung 8 dargestellt statische und dynamische Messungen durchgeführt.

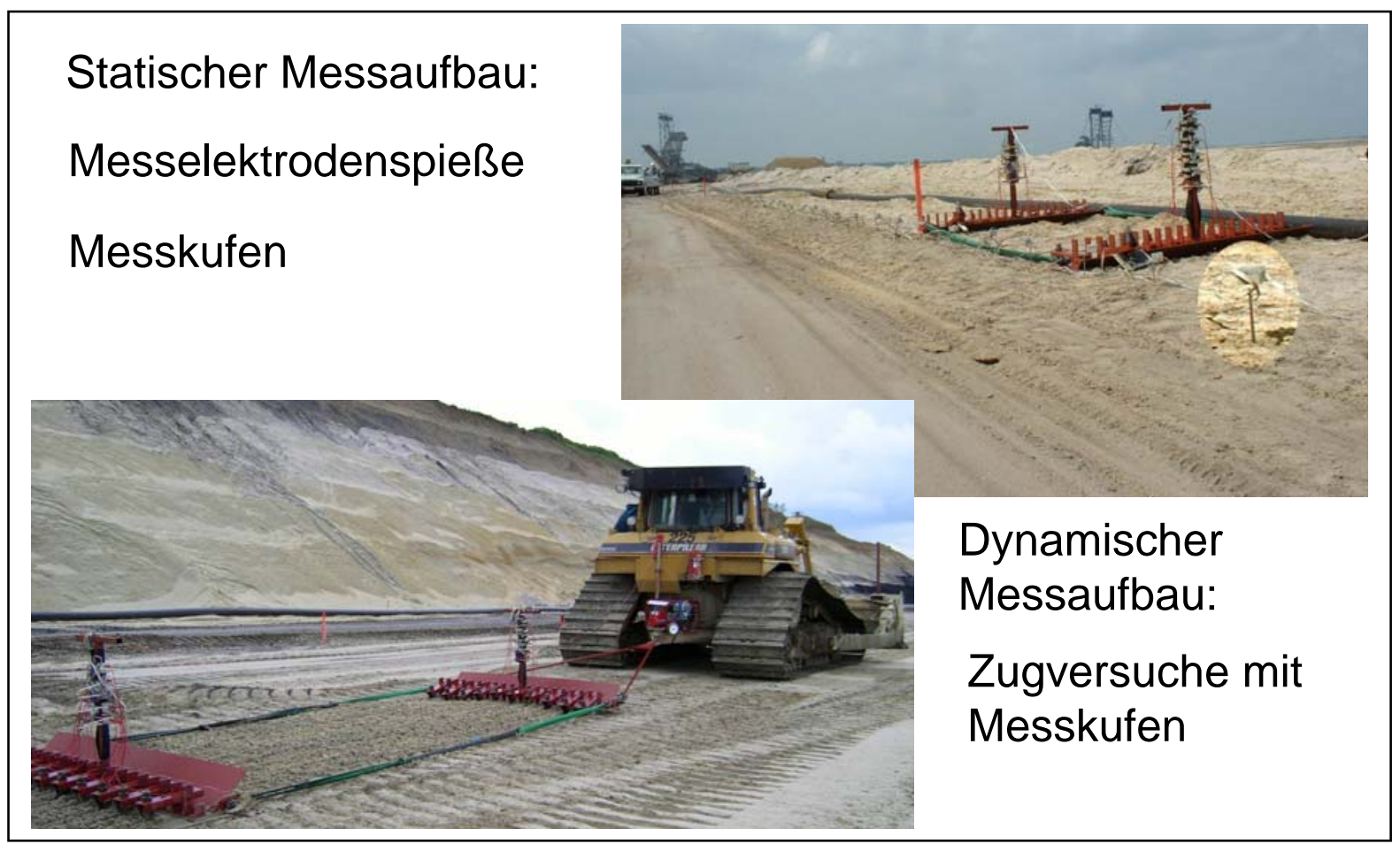

Abbildung 8: Messaufbau für statische und dynamische Geoelektrikversuche

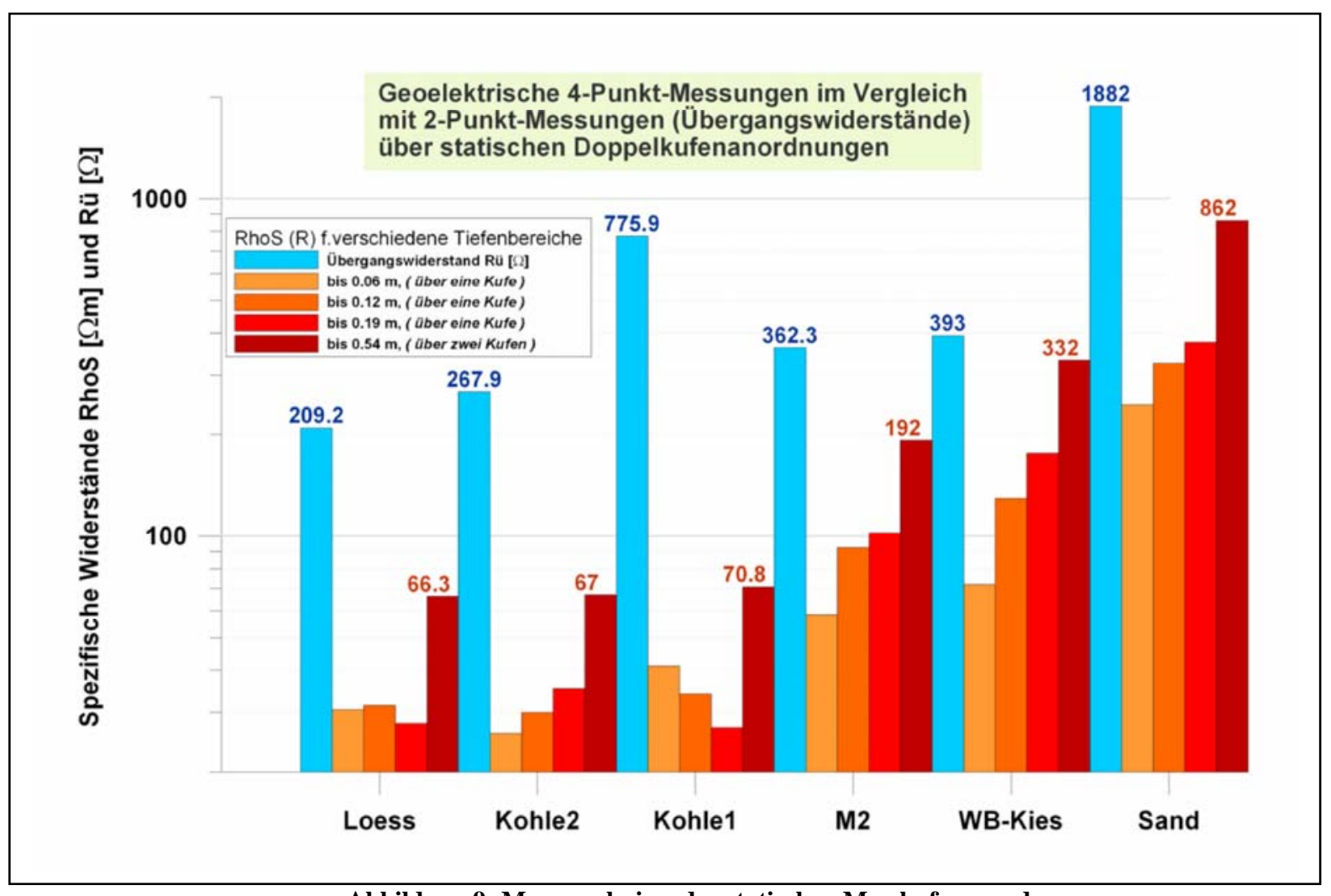

Abbildung 9: Messergebnisse der statischen Messkufenanordnung 
Die in Abbildung 9 dargestellten Ergebnisse der statischen Messungen zeigen die Unterscheidungsmöglichkeiten unterschiedlicher Materialarten über die geophysikalischen Parameter des spezifischen Widerstands. Beim dynamischen Schleppversuch entsteht in Abhängigkeit von der Geschwindigkeit der schleifenden Elektrode zum Untergrund ein materialspezifisches Schwanken des Übergangswiderstands, wodurch ein weiterer Parameter zur Materialklassifizierung gewonnen wird. In Abbildung 10 wird der deutliche Unterschied für das Beispiel Kohle und Sand ersichtlich. Aufgrund des geringeren Widerstands von Kohle gegenüber Sand sind die gemessenen Ströme größer. Auch die Amplitudenschwankungen der Messströme sind größer als beim Sand. Im Sand zeigt der Übergangswiderstand einen homogeneren Verlauf.

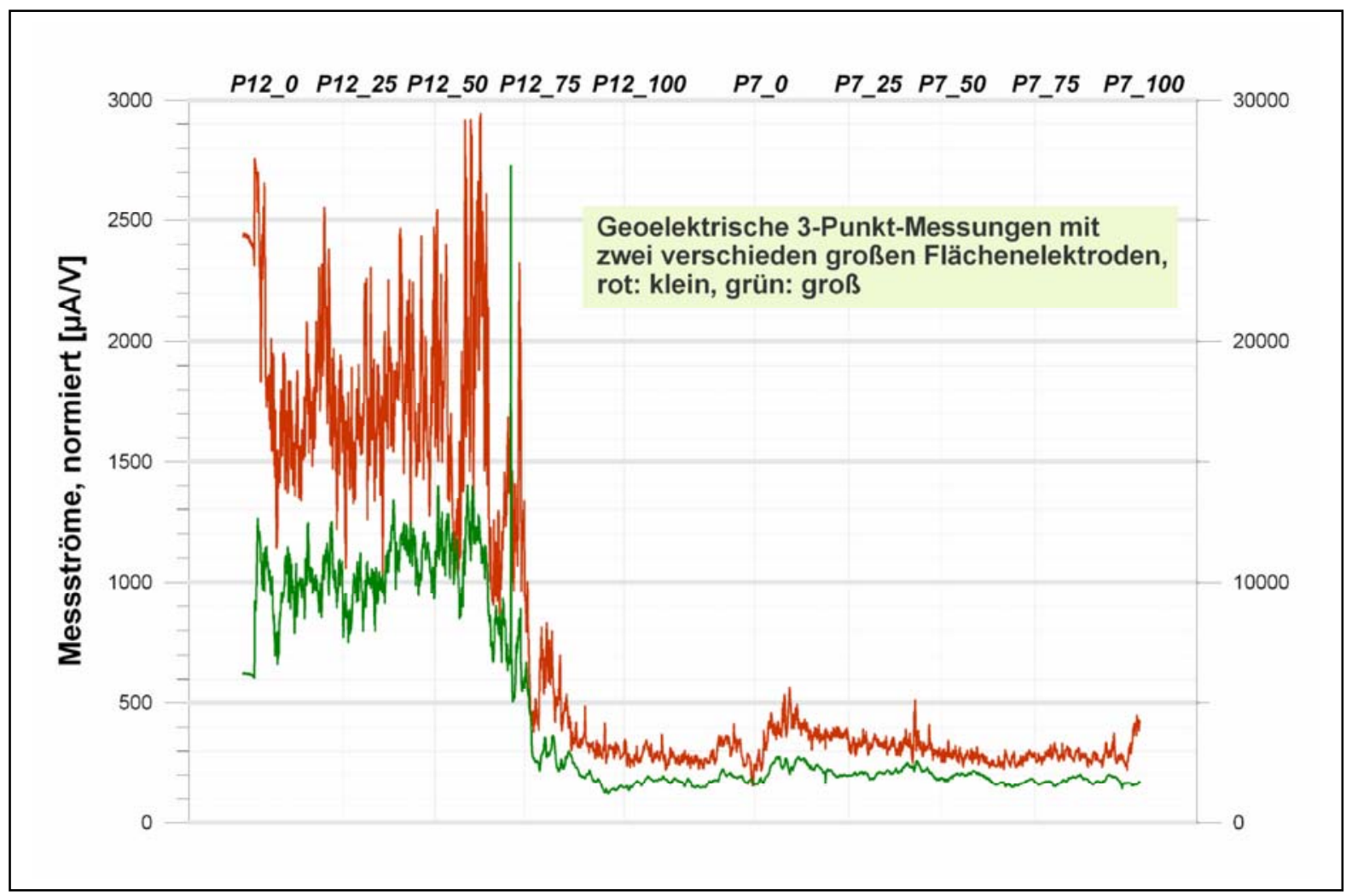

Abbildung 10: Messergebnis eines dynamischen Schleppkufenversuchs

Für die Geoelektrikversuche am Abbaustoß wurde derselbe Versuchsaufbau und -ablauf wie bei den Georadarversuchen gewählt, mit dem Unterschied, dass die auf dem Schaufelrücken platzierten Messelektroden im ständigen Kontakt zur Materialoberfläche über die Abbaustoßgeometrie geführt werden mussten. 


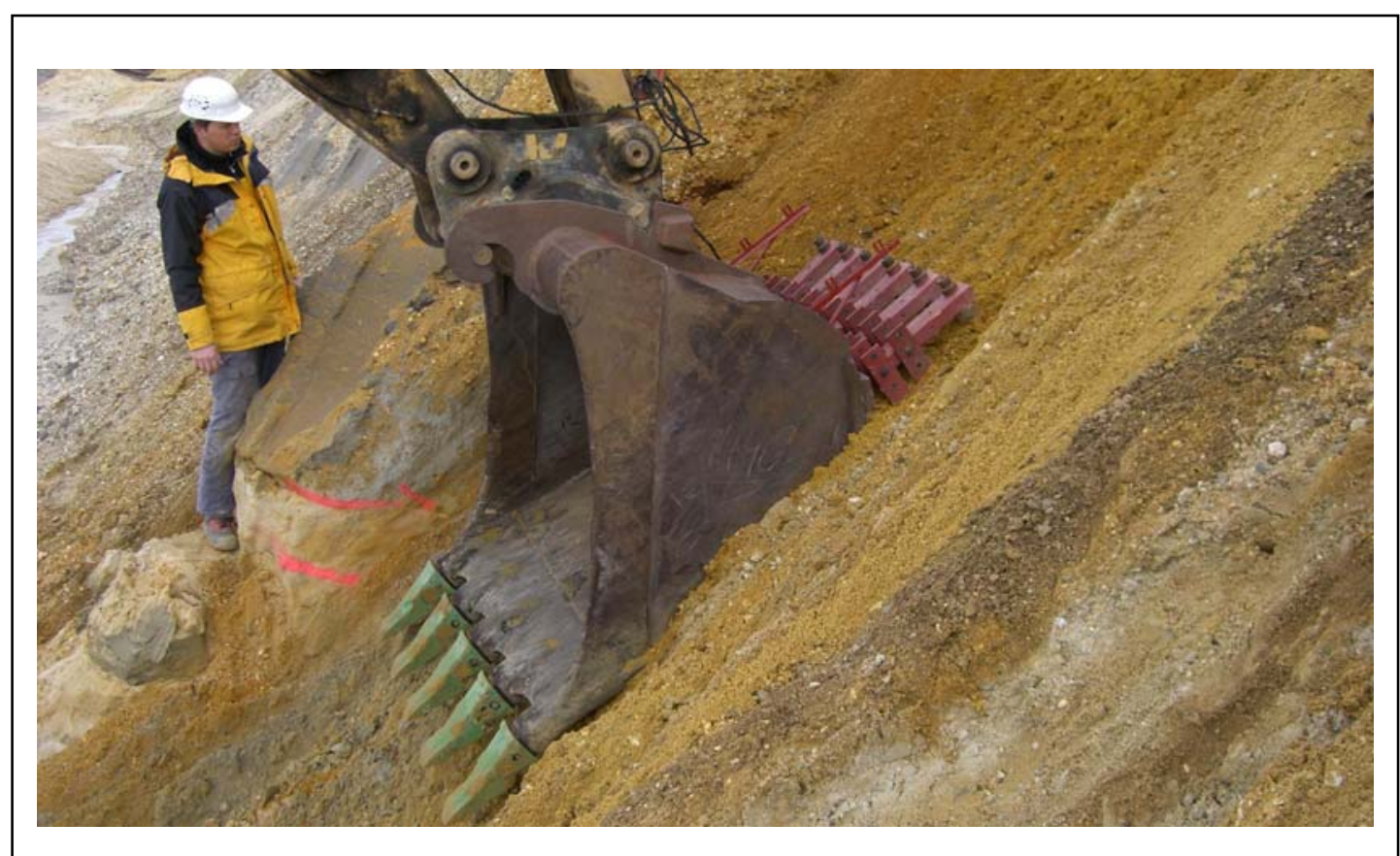

Abbildung 11: Messelektroden auf dem Schaufelrücken im Eingriff am AbbaustoßFazit und Ausblick

\subsection{Fazit}

Die während der Feldversuche untersuchten Bodenarten konnten bezüglich der Anforderungen zur Unterstützung des Abbaubetriebs mit ausreichender Genauigkeit klassifiziert und die dazwischen liegenden Schichtgrenzen detektiert werden. Bei der Detektion der Schichtgrenzen mit dem Georadar wurde eine Genauigkeit von +/$5 \mathrm{~cm}$ erreicht und somit die geforderte Auflösung von $+/-10 \mathrm{~cm}$ eingehalten. Die Kombination von Georadar und Geoelektrik ergänzt und unterstützt sich gegenseitig und führt zu einer leistungsfähigen Multisensorik. Für eine ausreichende Funktion in metallischer Umgebung am Schaufelradbagger ist eine Optimierung und Weiterentwicklung der Georadarantennen und der Geoelektrik erforderlich.

\subsection{Ausblick}

Die besondere Herausforderung liegt in der Integration der Sensoren am Schaufelradbagger. Aktuell werden hierzu Vorversuche zur Elektroden-, Antennen- und Messtechnikplatzierung an der Schaufel eines Schaufelradbaggers durchgeführt. Die Integration der Sensoriken darf sich nicht negativ auf den Betrieb oder die Standzeiten der einzelnen Abbaugeräte auswirken. Eine weitere Aufgabe ist die Optimierung und Weiterentwicklung der Georadarantennen und der Geoelektrik, um eine ausreichende Funktion im Schaufelrad und hohe Qualität der Rohdaten zu erzielen. In Abbildung 12 ist die Platzierung und Funktion der Sensorik am Schaufelradbagger dargestellt. Ein Gehäusedummy läuft bereits seit 5 Monaten ohne Beschädigungen im Abbaubetrieb auf dem Rücken einer Schaufel mit. Auf diesen Erfahrungen basierend wurde ein Gehäuse zur Aufnahme des Georadars und der Geoelektrik konstruiert, welches zunächst ohne die Sensoriken am Schaufelradbagger getestet werden soll. 


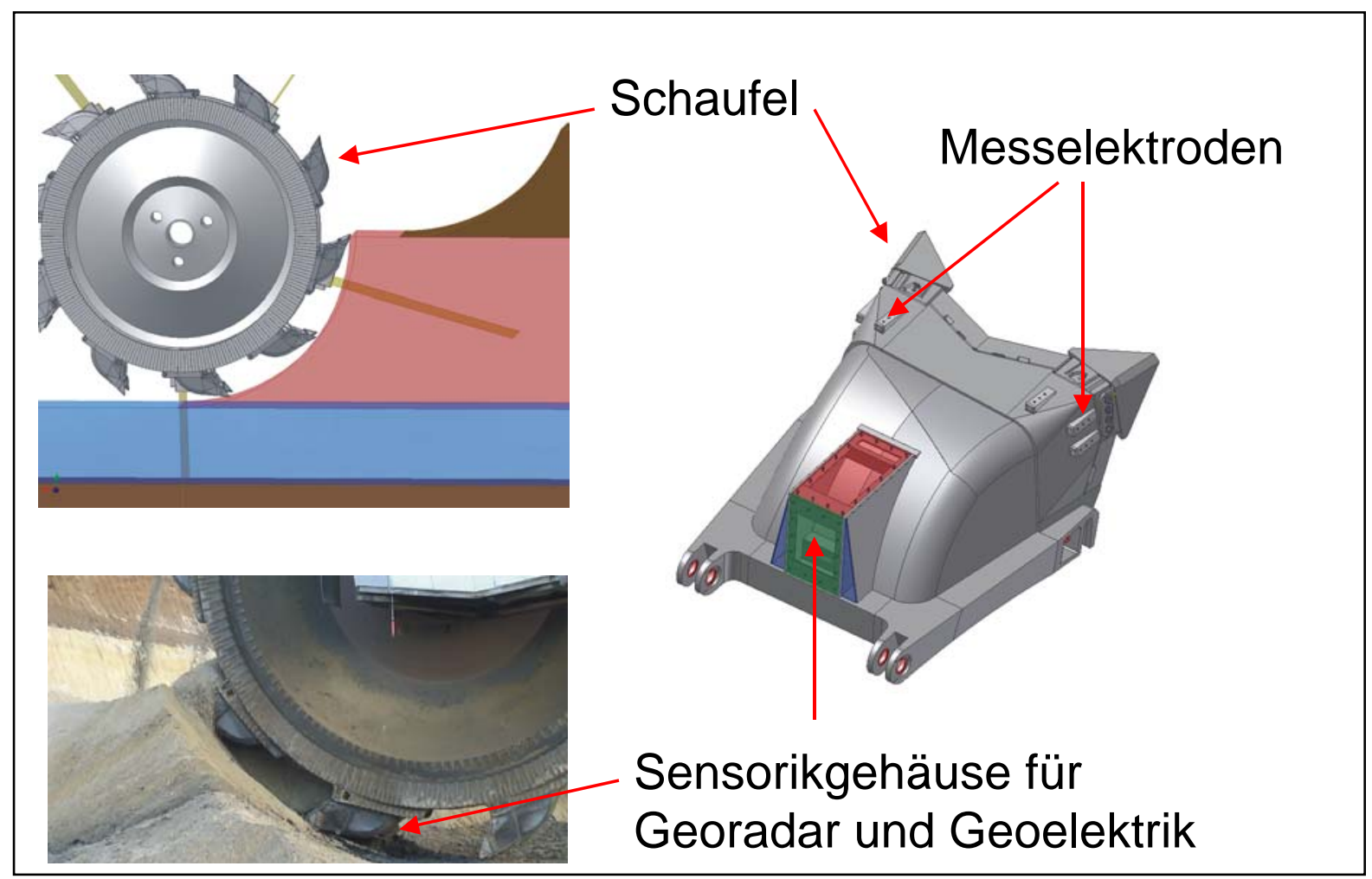

Abbildung 12: Integration der Sensorik in das Schaufelrad

Die weiterentwickelten Antennen werden zunächst in der Ebene und mit dem Hydraulikbagger am Abbaustoß getestet, um vor der Integration am Schaufelradbagger die Feinabstimmung zu realisieren. Für die spätere Darstellung in einem dreidimensionalen Modell müssen die Messdaten automatisch mit den absoluten Positionswerten verknüpft und ausgewertet werden. Mit Erreichung der Zielsetzung von SEMT wird ein weiterer Meilenstein in Richtung Vollautomatisierung und Prozessoptimierung im Tagebau erreicht.

\section{Literatur}

[ALT04]

[BER98]

[BRI03]

[DAN04 et.all] [DUR86]

[FOER01]

[GEO04]

[KLI03]

[KLI04]
Althaus, Paul Georg: Kontaktlose elektrische Verfahren zur Erkundung des oberflächennahen Untergrunds und deren Anwendung an Unstetigkeiten durch bergbaubedingte Bodenbewegungen, Verl. Glückauf, Essen, ISBN 3-7739-5996-6 *kart., 2004. Beres, Milan: Three-dimensional georadar imaging: quaternary sediments on the Rhine and Rhone River valleys, Switzerland, Swiss Fed. Inst. of Techn., Diss., Zurich, 1998.

Bristow, Charlie S.: Ground penetrating radar in sediments, Geological Soc. (GSL), London, ISBN 1-86239-131-9, 2003.

Daniels, David J.: Ground penetrating radar, ISBN 0-86341-360-9, 2004

Durst, Walter; Vogt, Werner: Schaufelradbagger, Trans Tech Publications, Clausthal-Zellerfeld, ISBN 0-87849-057-4, 1986.

Förster, Marc-Oliver; Hothan, Jürgen: Gültigkeit der mit dem "Ground Penetration Radar" (GPR) ermittelten Schichtdicken von Straßenbefestigungen, Bundesministerium für Verkehr, Bau- und Wohnungswesen, Bonn, ISBN 3-934458-62-9, 2001. Geohydraulikdata GdbR: Baugrund-Vorauserkundung in Echtzeit während des Tunnelvortriebs, 2004.

Klitzsch, N.:Characterizing unconsolidated sediments using the spectral inducted polarisation (SIP) method. EGS-AGU-EUG Joint Assembly, Nice, 2003.

Klitzsch, N.: Ableitung von Gesteinseigenschaften aus Messungen der spektral induzierten Polarisation (SIP) an Sedimentgesteinen, Dissertation, Universität Leipzig, 2004. 
[SCH00]

Schulz, Rüdiger: Angewandte Geophysik - neue Geräte und ihre Anwendungen, Bundesanstalt für Geowissenschaften und Rohstoffe, Schweizerbart, Stuttgart, ISBN 3-9801097-7-1, 2000.

[SIM05] Simpson, Fiona: Geophysik: Eine Einführung, Wiesbaden: Teubner, ISBN 3-51900464-X *Pb, 2005 